\title{
Comparative observation of the efficacy of simplified Descemet stripping endothelial keratoplasty and penetrating keratoplasty in treating bullous keratopathy
}

\author{
YINGXIN CHEN ${ }^{1}$, SHANSHAN SUN $^{1,2}$, MINGHONG GAO $^{1}$, QIMING LIU ${ }^{1}$ and ZIYUE WANG \\ ${ }^{1}$ Department of Ophthalmology, The General Hospital of Northern Theater Command, Shenyang, Liaoning 110840; \\ ${ }^{2}$ Department of Ophthalmology, The Third People's Hospital of Changzhou, Changzhou, Jiangsu 213001, P.R. China
}

Received April 23, 2020; Accepted July 17, 2020

DOI: $10.3892 /$ etm. 2020.9158

\begin{abstract}
The aim of the present study was to compare the clinical efficacy between simplified Descemet stripping endothelial keratoplasty (DSEK) and penetrating keratoplasty (PKP) in the treatment of patients with bullous keratopathy (BK). A cohort of 65 patients (65 eyes) with BK recruited between December 2002 and June 2018 was divided into two groups according to the treatment they received: The simplified DSEK group $(n=38)$ and the PKP group $(n=27)$. The best-corrected visual acuity (BCVA) during the follow-up at 1, 3, 6 and 12 months and postoperative complications were all recorded. Furthermore, the graft survival rate after 1 year was recorded. The mean BCVA in the simplified DSEK group was significantly better than that in the PKP group after 1 , 3, 6 and 12 months $(\mathrm{P}<0.05)$. Furthermore, the 1-year graft survival rate in the simplified DSEK group (91.2\%) was significantly higher than that in the PKP group (70.4\%; $\mathrm{P}=0.039)$. A total of 13 eyes $(34.21 \%)$ in the simplified DSEK group and 11 eyes $(40.74 \%)$ in the PKP group were diagnosed with glaucoma; there was no significant difference between the rate of glaucoma diagnosis between the two groups $(\mathrm{P}=0.591)$. Graft rejection was observed in 5 eyes $(13.16 \%)$ of the simplified DSEK group and 8 eyes $(29.63 \%)$ of the PKP group and the rate of graft rejection did not differ significantly between the groups $(\mathrm{P}=0.279)$. Graft infection occurred in 1 eye $(2.63 \%)$ in the simplified DSEK group and 6 eyes $(22.22 \%)$ in the PKP group. Simplified DSEK achieved better visual acuity and longer graft survival rates than PKP. The incidence of postoperative secondary glaucoma, graft rejection and graft infection after simplified DSEK was lower than that in the PKP group,
\end{abstract}

Correspondence to: Dr Minghong Gao, Department of Ophthalmology, The General Hospital of Northern Theater Command, 83 Wenhua Road, Shenhe, Shenyang, Liaoning 110840, P.R. China E-mail: gaominghong88@yeah.net

Key words: simplified Descemet stripping endothelial keratoplasty, bullous keratopathy, penetrating keratoplasty but only the incidence of graft infection was significantly different.

\section{Introduction}

Bullous keratopathy (BK) is amongst the most common causes of corneal decompensation, frequently requiring corneal transplantation (1). Endothelial keratoplasty (EK) has become an effective alternative to penetrating keratoplasty (PKP) for treating BK caused by a dysfunctional endothelium (2). Compared to PKP, the application of EK provides more rapid recovery of vision, minimized induction of astigmatism, and, more importantly, better maintenance of the integrity of the globe $(3,4)$.

At present, the major techniques of EK include Descemet's stripping endothelial keratoplasty (DSEK)/Descemet's stripping with automated endothelial keratoplasty (DSAEK) and Descemet's membrane endothelial keratoplasty (DMEK) (5). Due to the inapplicability of DMEK in patients with serious edematous stroma or aphakic eyes, which have previously undergone vitrectomy, DSAEK remains in use as the primary procedure for EK (2). In DSAEK, creating thinner grafts is thought to improve visual outcomes and anatomic success; however, the creation of thinner donor tissue is technically challenging (3).

A major problem with the use of DSEK is the intricate manual preparation required for the lamellar graft, frequently resulting in irreparable damage to the material intended for transplantation $(6,7)$. The implantation method used at our department has been simplified. For the simplified DSEK, the graft may be successfully implanted through a transparent limbal incision without the use of special instruments and the surgical incision is small with rapid recovery. The present study aimed to evaluate the difference in the clinical efficacy between the simplified DSEK method and PKP for BK.

\section{Materials and methods}

Patients. A total of 65 patients (65 eyes) with BK between December 2002 and June 2018 from the General Hospital of Northern Theater Command (Shenyang, China) were retrospectively included in the present study. Patients who received modified 
simplified DSEK were assigned to the modified simplified DSEK group $(\mathrm{n}=38)$ and patients who received PKP were designated as the PKP group $(n=27)$. The present study was approved by the Ethics Committee of the General Hospital of Shenyang Military Region and all patients provided written informed consent.

The inclusion criteria were as follows: i) Patients with BK; ii) underwent their first corneal transplantation; iii) $\geq 18$ years and $\leq 90$ years of age and iv) a follow-up period of $\geq 1$ year. The following exclusion criteria were the presence of fundus lesions that affected postoperative vision, including macular degeneration, fundus hemorrhage or optic nerve atrophy.

The donor corneas were all from healthy and fresh eyes of deceased domestic donors. Preoperative corneal examination of the donor was completed as follows: Slit-lamp microscope examination was performed to confirm the transparency and smoothness; corneal endothelium was used to observe the morphology and clarity of endothelial cells, and the density of endothelial cells was required to be $>2,000 / \mathrm{mm}^{2}$.

Surgical methods. The combined surgical methods of the simplified DSEK and PKP groups are presented in Tables I and II, respectively. All surgeries were performed by the same corneal transplant surgeon. Pilocarpine eye drops (5 min/administration) were given 6 times to patients undergoing corneal transplantation. For patients undergoing combined surgery, pupil dilatation was performed according to the surgical conditions, and then, $0.1 \mathrm{ml}$ of carbachol injection was given to shrink the pupil for subsequent keratoplasty.

Prior to the operation, the patients were given routine treatments, including cleaning the eyelid margin, eyelash trimming, conjunctival sac irrigation and lacrimal tract irrigation. All patients were given topical anesthesia with promevacaine hydrochloride eye drops (Erkeine); 2\% lidocaine injection was used for sufficient retrobulbar and peribulal block anesthesia. Oorbicularis oculi muscle anesthesia was also performed for the PKP group.

Simplified DSEK. The graft bed was prepared. First, a mark on the corneal epithelium was made with a suitable trephine. A paracentesis knife was used to make a 5-mm-wide transparent limbal main incision at the clock position of 12:00 to enter the anterior chamber and the viscoelastic agent was injected. Two transparent limbal auxiliary incisions at the clock positions of 3:00 and 9:00 were made. After removing the hook and scraping the elastic layer and endothelial cell layer along the marking ring, the viscoelastic agent in the anterior chamber was then rinsed and sterile air was injected to press the graft bed to form the anterior chamber.

The corneal endothelial graft was then prepared as follows: A vacuum negative pressure trephine with different diameters was rotated perpendicular to the donor cornea surface to make the anterior deep lamellar corneal slice, with a thickness of $400-450 \mu \mathrm{m}$, and the anterior deep lamellar corneal slice was removed. The donor posterior lamellar corneal slice was cut in a 1-2 $\mathrm{mm}$ ring along the outer edge of the sclera, the inner skin was placed on the special corneal incision pillow, and then the endothelial graft with partial posterior stroma was drilled with a trephine used for the graft bed.

The graft was implanted using the slide method (8) as follows: A plastic slide with a width of $\sim 4 \mathrm{~mm}$ was placed into the anterior chamber through the upper main incision. After dripping an appropriate quantity of viscoelastic agent on the surface of the slide outside the incision, the graft endothelium was laid on the slide remaining outside the incision and the graft was then moved into the anterior chamber with a self-made 1-ml syringe crochet needle. A small quantity of lactated Ringer's solution was injected into the anterior chamber to make the graft expand appropriately. The main incision was intermittently sutured with 10/0 suture for 3 stitches. Ringer's lactate solution was re-injected from the side incision into the anterior chamber to fully expand the graft. The position of the graft was adjusted and sterile air was injected into the anterior chamber to make the graft closely attach to the graft bed. The surface gas injection was successful when a 'double ring sign' was observed. Finally, iris restorer was placed firmly on the corneal surface to drain the excess liquid and gas between the graft and the graft bed, which was conducive to the close attachment of the graft and the graft bed. After surgery, all patients were treated with tobramycin and dexamethasone eye ointment (Dianshu).

The patient was instructed to lie in the supine position within postoperative $24 \mathrm{~h}$ and the gas was placed directly under the implant, which was conducive for better adhesion of the corneal endothelium and increased the success rate of implant transplantation.

Phacoemulsification + intraocular lens (IOL) implantation + simplified DSEK. First, a transparent limbal tunnel incision was made at the clock position of 12:00 with a stab knife, followed by routine phacoemulsification + IOL implantation. The main incision was expanded to $5 \mathrm{~mm}$ and simplified DSEK was then performed.

Separation of the chamber angle from the anterior synechia + coreoplasty + phacoemulsification + IOL implantation + simplified DSEK. A stab knife was used to make a 5-mm-wide transparent limbal main incision at the clock position of 12:00 and the viscoelastic agent was injected. A transparent auxiliary incision was made near the anterior synechia. Capsular scissors were used to cut the adhesive iris and the iris restorer was used to separate the adhesive chamber angle. The iris was cut and sutured to make the pupil circular. Routine phacoemulsification + IOL implantation and simplified DSEK were then performed.

$I O L$ reduction + coreoplasty + simplified DSEK. The main and auxiliary corneal limbal incisions were made at the 10:00 and 2:00 clock positions, respectively, and then, the iris restorer was used to enter the anterior chamber through the main incision to restore the intraocular lens to the normal position and restore the iris shape. Simplified DSEK was then performed.

Phacoemulsification + IOL implantation + iris root resection + simplified DSEK. The transparent limbal main incision was made at the clock position of 12:00 to enter the anterior chamber and the viscoelastic agent was injected. The iris restorer was used to separate the adhesive iris and the bulbar conjunctiva was cut open to make the scleral flap. The $8 / 0$ suture was inserted into the anterior chamber from the clock position of 12:00 and was perforated from the detached iris root under 
Table I. Operation methods in the simplified DSEK group $(n=38)$.

Operation method

Cases, n $(\%)$

Simplified DSEK

Phacoemulsification + IOL implantation + simplified DSEK

Coreoplasty + simplified DSEK

IOL reduction + coreoplasty + simplified DSEK.

Separation of chamber angle from anterior synechia + coreoplasty + Phacoemulsification + IOL

implantation + simplified DSEK

Phacoemulsification + IOL implantation + iris root resection + simplified DSEK

DSEK, Descemet stripping endothelial keratoplasty; IOL, intraocular lens.

Table II. Operation methods in the PKP group $(n=27)$.

\begin{tabular}{lc}
\hline Operation method & Cases, n (\%) \\
\hline PKP & $22(81.5)$ \\
Phacoemulsification + IOL implantation + PKP & $2(7.4)$ \\
IOL implantation + PKP & $2(7.4)$ \\
Trabeculectomy + PKP & $1(3.7)$ \\
\hline
\end{tabular}

PKP, penetrating keratoplasty; IOL, intraocular lens.

the scleral flap. The detached iris root was sutured using mattress suture, the scleral flap was reset and the scleral flap and conjunctiva were sutured with $8 / 0$ absorbable suture.

$P K P$. The operational procedure was performed as described previously (9). The graft bed was prepared as follows: A suitable trephine was selected perpendicular to the surface of the cornea and rotated clockwise into the cornea to drill the graft bed.

Corneal scissors were used to cut $2 \mathrm{~mm}$ along the limbus sclera to obtain the donor cornea and the inner skin was placed on the special corneal incision pillow. A trephine with a diameter of $>0.25 \mathrm{~mm}$ was used to drill the donor cornea to obtain the graft. The 10/0 suture was first performed intermittently at the 3,6,9 and 12 o'clock positions to fix the graft and suturing was then performed for a total of 12-16 stitches to bury the knot. A total of $50 \mu 1$ of balanced saline solution was injected into the anterior chamber. All patients were treated with tobramycin dexamethasone eye ointment and monocular compression bandaging, and the eye condition and the intraocular pressure were closely monitored.

If the cataracts were combined, extracapsular cataract extraction + IOL or phacoemulsification + IOL implantation was performed, and the graft and graft bed were then sutured intermittently.

Postoperative management. For the simplified DSEK group, all patients were placed in a supine position for $24 \mathrm{~h}$ after surgery. Patients were given systemic ocular hypotensive drugs (20\% mannitol, $250 \mathrm{ml}$ b.i.d.) the day after surgery. Eyes were opened on the day after surgery and anti-infection and anti-rejection treatment were administered. During the postoperative follow-up, the medication regimen was adjusted according to the recovery of the operated eye. The sutures were removed at 3-6 months after the operation.

For the PKP group, a monocular compression bandage was applied for 3 days following surgery. If the anterior chamber was well formed, the eyes were opened and anti-infection and anti-rejection treatment were given. During the postoperative follow-up, the medication regimen was adjusted according to the recovery of the operative eye. The changes in the intraocular pressure of the surgical eye were observed. The eye sutures were removed 6-12 months after surgery.

Postoperative medication. All patients were given levofloxacin eye drops (t.i.d.) for 1 year, sodium hyaluronate eye drops (b.i.d.) for 1 year, tobramycin dexamethasone eye ointment (q.d.) for 6 months, tacrolimus (b.i.d.) for 1 year and prednisolone acetate eye drops ( 6 times a day) for 6 months. The combined anti-rejection therapy of Belit was administered after epithelialization was complete.

Follow-up. The patients were all followed up on postoperative weeks 1 and 2, and after 1, 3, 6, 12 and 24 months. At each follow-up point, slit-lamp microscopy was used to observe the attachment of eye grafts to graft beds. Intraocular pressure (IOP), average best-corrected visual acuity (BCVA), corneal opacity, postoperative complications and corneal graft survival rates were determined. When the BCVA was measured in Snellen, it was converted to a logarithm of the minimum angle of resolution ( $\log \mathrm{MAR})$ to facilitate statistical analysis. Counting fingers, hand movements and perception of light were assigned the arbitrary values of 1.60, 1.90 and 2.20 $\log$ MAR, respectively. The same assumptions were made previously (10).

Secondary glaucoma was defined as follows: IOP or estimated IOP $\geq 24 \mathrm{mmHg}$ and requires intervention with IOP-lowering medication, postoperative IOP is $10 \mathrm{mmHg}$ higher than the preoperative IOP, or the IOP cannot be controlled by drugs and surgical treatment is required (11-14). Primary transplant failure refers to transparent persistent irreversible corneal edema after surgery (15).

Postoperative implant displacement and detachment. Displacement refers to the partial separation of the graft from 
Table III. Baseline information of the patients.

\begin{tabular}{|c|c|c|c|c|}
\hline Item & Simplified DSEK (n=38) & $\operatorname{PKP}(n=27)$ & $\chi^{2 / t}$ & P-value \\
\hline Age (years) & $68.03 \pm 10.82$ & $66.26 \pm 9.80$ & 0.674 & 0.503 \\
\hline Male, n (\%) & $22(57.89)$ & $14(51.85)$ & 0.233 & 0.629 \\
\hline Preoperative diagnosis, $\mathrm{n}(\%)$ & & & 3.944 & 0.414 \\
\hline PBK & $29(76.32)$ & $23(85.19)$ & & \\
\hline Fuchs & $3(7.89)$ & $3(11.11)$ & & \\
\hline Viral endocorneal dermatitis & $4(10.53)$ & 0 & & \\
\hline ICE syndrome & $1(2.63)$ & $1(3.70)$ & & \\
\hline Trauma & $1(2.63)$ & 0 & & \\
\hline History of glaucoma, n (\%) & $15(39.47)$ & $6(22.22)$ & 2.148 & 0.143 \\
\hline Lens state, $\mathrm{n}(\%)$ & & & 0.375 & 0.829 \\
\hline Artificial lens & $27(71.05)$ & $21(77.78)$ & & \\
\hline Lens & $9(23.69)$ & $5(18.52)$ & & \\
\hline Absence of lens & $2(5.26)$ & $1(3.70)$ & & \\
\hline BCVA (logMAR) & $1.81 \pm 0.24$ & $1.83 \pm 0.23$ & -0.351 & 0.727 \\
\hline
\end{tabular}

Values are expressed as the mean \pm standard deviation or $\mathrm{n}(\%)$. PBK, pseudophakic bullous keratopathy; ICE syndrome, iridocorneal endothelial syndrome; BCVA, best-corrected visual acuity; $\log \mathrm{MAR}$, logarithm of the minimum angle of resolution.

the graft bed. Detachment refers to the complete separation of the graft from the graft bed, or even dislocation into the anterior chamber (16).

Statistical analysis. Statistical analysis was performed using SPSS version 24.0 (IBM Corp.). Values are expressed as the mean \pm standard deviation or $\mathrm{n}(\%)$. Age and preoperative BCVA were compared using Student's t-test. Preoperative diagnosis, lens state, history of glaucoma, gender and incidence of postoperative complications were compared using $\chi^{2}$ tests. Postoperative visual acuity was compared using the Mann-Whitney U-test. Kaplan-Meier curves were generated to analyze the graft survival rate of the two groups. Log-rank test was used for comparison between the two Kaplan-Meier curves. $\mathrm{P}<0.05$ was considered to indicate a statistically significant difference.

\section{Results}

Baseline characteristics. A total of 65 patients (65 eyes) with bullae keratopathy were included in this study, including 36 male eyes and 29 female eyes. The mean age was $67.3 \pm 10.4(45-84)$ years. There were no significant differences in the preoperative basic characteristics including age, sex, preoperative diagnosis, history of glaucoma, lens state and BCVA between the two groups (all $\mathrm{P}>0.05$; Table III).

BCVA. As presented in Table IV, the average BCVA logMAR at 1, 3, 6 and 12 months in the simplified DSEK group was significantly lower than that in the PKP group $(\mathrm{P}<0.05)$. The BCVA interval distribution at the last follow-up is presented in Table $\mathrm{V}$ and the results indicated that the visual acuity of the simplified DSEK group was significantly better than that in the PKP group.
Graft survival rate at the last follow-up. Corneal graft changes within 1 year after simplified DSEK and PKP in representative cases are presented in Figs. 1 and 2, respectively. At the last follow-up at 1 year after surgery, the graft survival rate in the simplified DSEK group was significantly higher compared with that in the PKP group (91.17 vs. $70.37 \%, \mathrm{P}=0.039$ ). Kaplan-Meier curves for graft survival in the two groups are provided in Fig. 3.

Postoperative complications and management. As presented in Table VI, 13 eyes (34.21\%) in the simplified DSEK group and 11 eyes (40.74\%) in the PKP group developed secondary glaucoma; this difference was not statistically significant $(\mathrm{P}=0.591)$. A total of 8 eyes $(61.54 \%)$ in the simplified DSEK group were treated with antihypertensive drugs and the IOP dropped to within the normal range. A total of 3 eyes (23.08\%) developed drug dependence. In addition, 2 eyes (15.38\%) received anti- glaucoma surgery, 1 for glaucoma drainage implant surgery and 1 for glaucoma valve implant surgery. In the PKP group, 4 eyes $(36.36 \%)$ were treated with antihypertensive drugs and the IOP dropped within the normal range. In addition, 5 eyes $(45.45 \%)$ developed drug dependence and 2 eyes (18.18\%) received anti-glaucoma surgery, 1 for glaucoma trabeculectomy and 1 for glaucoma valve implant surgery.

As presented in Table VI, graft rejection was observed in 5 eyes $(13.16 \%)$ from the simplified DSEK group and 8 eyes (29.63\%) from the PKP group; this difference was not statistically significant $(\mathrm{P}=0.279)$. Immunological rejection occurred in 5 eyes $(13.16 \%)$ of the simplified DSEK group and 8 eyes (29.63\%) of the PKP group. A total of 3 eyes from the simplified DSEK group were subjected to anti-rejection treatment and 2 eyes had progressive development-induced transplantation failure, 1 of which was treated with PKP 12 months after the operation. In addition, 4 eyes from the PKP group were subjected to anti-rejection treatment and 4 eyes exhibited 
Table IV. Postoperative mean best-corrected visual acuity (logarithm of the minimum angle of resolution) compared between the two groups.

\begin{tabular}{llrr}
\hline Time-point & Simplified DSEK & PKP & Z-value \\
\hline Following surgery (months) & & & \\
1 & $0.95 \pm 0.41(\mathrm{n}=38)$ & $1.10 \pm 0.34(\mathrm{n}=27)$ & -2.421 \\
3 & $0.81 \pm 0.54(\mathrm{n}=38)$ & $1.04 \pm 0.38(\mathrm{n}=27)$ & -3.499 \\
6 & $0.67 \pm 0.63(\mathrm{n}=37)$ & $0.99 \pm 0.52(\mathrm{n}=26)$ & -3.196 \\
12 & $0.62 \pm 0.72(\mathrm{n}=35)$ & $1.02 \pm 0.65(\mathrm{n}=25)$ & -3.403 \\
\hline
\end{tabular}

Values are expressed as the mean \pm standard deviation. DSEK, Descemet stripping endothelial keratoplasty; PKP, penetrating keratoplasty.

Table V. BCVA interval distribution of the simplified DSEK group and the PKP group at 1-year follow-up.

\begin{tabular}{lcr}
\hline BCVA (logMAR) & Simplified DSEK $(\%)$ & PKP $(\%)$ \\
\hline$\geq 1.0$ & 17.14 & 28.00 \\
$0.7-1.0$ & 8.75 & 12.00 \\
$>0.4, \leq 0.7$ & 11.43 & 56.00 \\
$\leq 0.4$ & 62.86 & 4.00 \\
\hline
\end{tabular}

BCVA, best-corrected visual acuity; logMAR, logarithm of the minimum angle of resolution; DSEK, Descemet stripping endothelial keratoplasty; PKP, penetrating keratoplasty.

progressive development-induced transplantation failure. Graft rejection changes of two representative cases after simplified DSEK and PKP are presented in Figs. 4 and 5.

As indicated in Table VI, the graft infection rate in the simplified DSEK group was significantly lower than that in the PKP group $(\mathrm{P}=0.012)$. Only 1 eye $(2.63 \%)$ in the simplified DSEK group had a secondary bacterial infection at 1 year after the operation. The infection was under control after anti-infection treatment, but the visual function was lost. A total of 6 eyes (22.22\%) from the PKP group had secondary graft infection after surgery, including 4 eyes with bacterial infection, 1 eye with fungal infection and 1 eye with viral infection. In the 4 eyes with bacterial infection, the infection was controlled after anti-infective treatment in 2 eyes, but the corneal graft lost its transparency. The other 2 eyes received PKP and eyeball removal, respectively. In 1 eye with a fungal infection, the infection was controlled and the cornea recovered its transparency after the lesion was scraped and antifungal treatment was applied. In 1 eye with viral infection, the infection was also controlled following antiviral treatment.

\section{Discussion}

The advantages of the simplified DSEK operation implemented at our department compared with conventional DSEK are as follows: i) The implantation incision was a transparent limbal incision with a width of $\sim 5.0 \mathrm{~mm}$, which resulted in smaller surgical trauma and faster recovery; ii) the graft may be implanted into the anterior chamber using a special 1-ml syringe without suture traction and special traction instruments; and iii) a plastic slide we used was a flexible soft material, which caused little injury to the endothelial cells during implantation.

In the present study, the overall average BCVA in the simplified DSEK group was significantly improved compared with that in the PKP group. Kosker et al (15) indicated that the visual acuity of patients in the simplified DSEK group remained stable after 3 months, while the visual acuity of the patients in the PKP group fluctuated throughout the follow-up. The authors assumed that the visual acuity fluctuations after PKP were primarily due to non-stop treatment of corneal astigmatism. Similar results were obtained in the present study. In the simplified DSEK group, the BCVA had gradually stabilized at 3 months after the operation with a small fluctuation range, while the average postoperative BCVA of the PKP group were unstable. For these reasons, it was speculated that corneal graft sutures require constant removal of the sutures, resulting in large fluctuations in corneal astigmatism and curvature. In addition, long-term use of local immunosuppressants and glucocorticoid eye drops cause ocular surface immunity to decrease, and therefore, grafts are prone to secondary infections, which results in failure.

In the present study, $35.48 \%$ of patients in the simplified DSEK group and no patients in the PKP group had an average BCVA of $>0.5$, which was lower than that in a previous study by Kosker et al (15), where the average BCVA was $>0.5$ in $80 \%$ of the patients treated with simplified DSEK and $46 \%$ of the patients in the PKP group. It was speculated that this was primarily associated with the preoperative diagnosis, as the patients in the study performed by Kosker et al (15) had Fuchs corneal dystrophy; however, the majority of the patients in the present study had pseudophakic BK. In the present study, the large difference in the postoperative visual acuity between the two groups was considered to be associated with the large astigmatism of the cornea caused by sutures after PKP, while the overall visual acuity after simplified DSEK was better because there were only a few sutures in the clear corneal margin after the surgery and the anterior corneal surface curvature was not damaged during the surgery.

The graft survival rate of the simplified DSEK group in the present study (91.2\%) was similar to that in a previous study (95.5\%); however, in the PKP group, the graft survival rate was significantly lower (70.4\%) compared with that in the previous study (91.4\%) (17). This may be due to the increased incidence of corneal transplantation failure caused by delayed treatment 


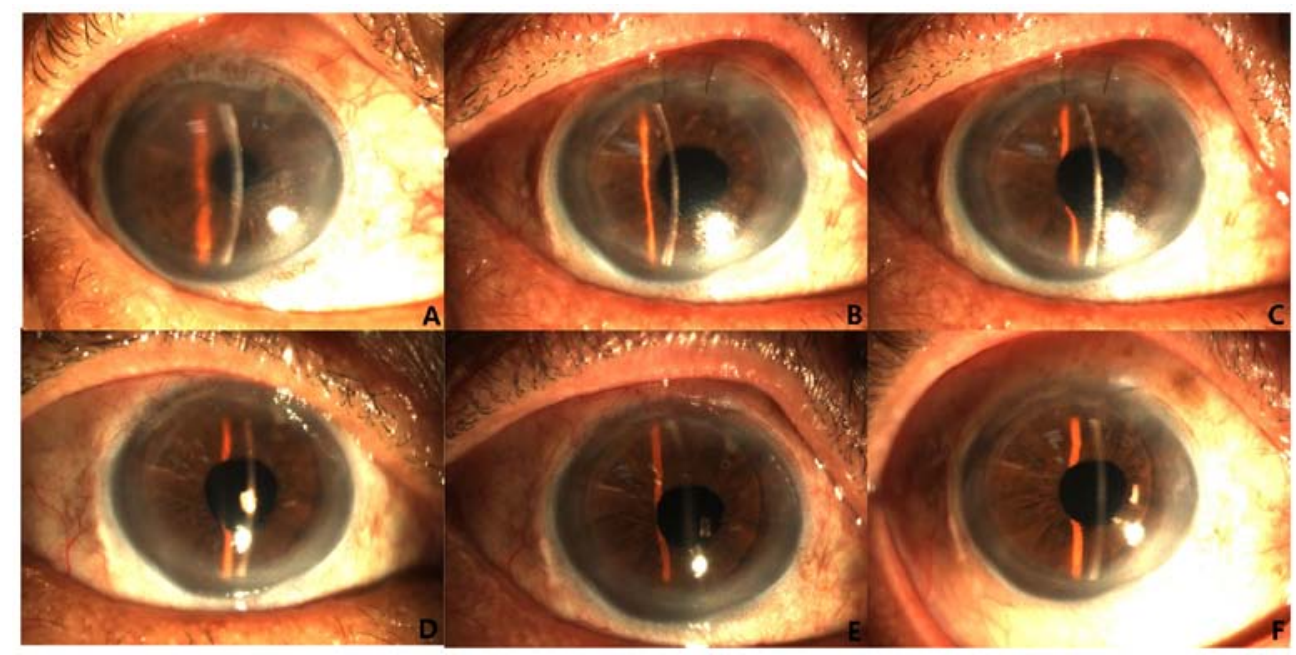

Figure 1. Corneal graft changes of a 78-year-old male patient within 1 year following simplified Descemet stripping endothelial keratoplasty. (A) Corneal mist edema and posterior elastic layer folds were observed prior to the operation. Corneal graft at (B) 1 week, (C) 1 month, (D) 3 months, (E) 6 months and (F) 1 year following surgery.

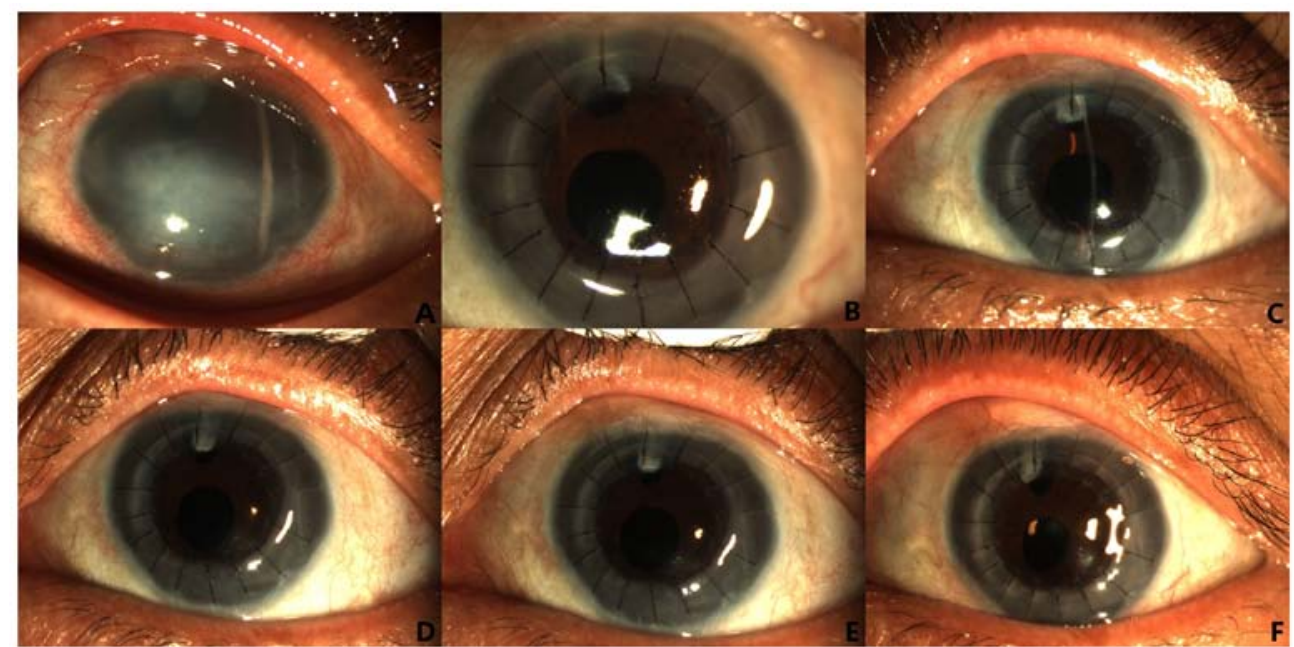

Figure 2. Corneal graft changes of a 63-year-old female patient within 1 year following penetrating keratoplasty. (A) Thickened corneal edema was observed prior to the operation. Corneal graft at (B) 1 month, (C) 3 month, (D) 4 months, (E) 6 months and (F) 1 year following surgery.

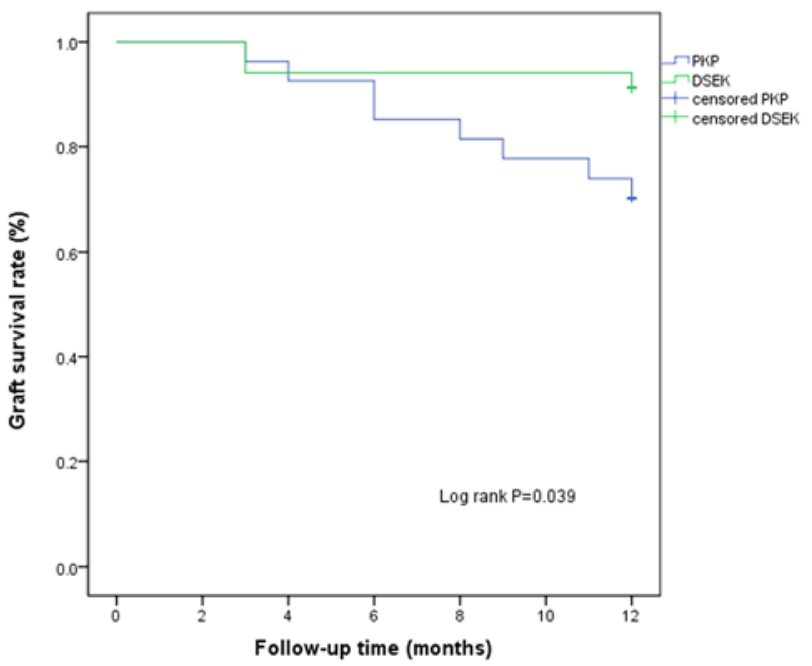

Figure 3. Kaplan-Meier curves for the graft survival rate in the two groups. DSEK, Descemet stripping endothelial keratoplasty; PKP, penetrating keratoplasty. of postoperative complications, or instead, it may be due to the small number of cases in the PKP group. In addition, the incidence of graft rejection and graft infection in the PKP group was significantly higher than that in the simplified DSEK group and these two complications tend to lead to secondary graft failure (18).

Glaucoma is one of the most common complications after corneal transplantation and the degree of damage is generally linked to the surgical method, particularly PKP, DSEK and Boston artificial corneal transplantation (19). The postoperative incidence was $33.33 \%$, which was consistent with that of previous studies $(13,19,20)$. The incidence of postoperative glaucoma in the DSEK group of the present study was $34.21 \%$, which was higher than that in previous studies, which reported incidences of $0-15 \%(21,22)$, but was similar to that in other studies that indicated that the incidence of glaucoma after DSEK may be as high as $35-39 \%(23,24)$. The high incidence of glaucoma in the present study may have been due to the requirement to inject 
Table VI. Postoperative complications compared between the two groups.

\begin{tabular}{lcrrr}
\hline Complication & Simplified DSEK $(\mathrm{n}=38)$ & PKP $(\mathrm{n}=27)$ & $\chi^{2}$ & P-value \\
\hline Secondary glaucoma & $13(34.21)$ & $11(40.74)$ & 0.289 & 0.591 \\
Graft rejection & $5(13.16)$ & $8(29.63)$ & 1.171 & 0.279 \\
Graft infection & $1(2.63)$ & $6(22.22)$ & 6.304 & 0.012 \\
\hline
\end{tabular}

Values are expressed as n (\%). DSEK, Descemet stripping endothelial keratoplasty; PKP, penetrating keratoplasty.

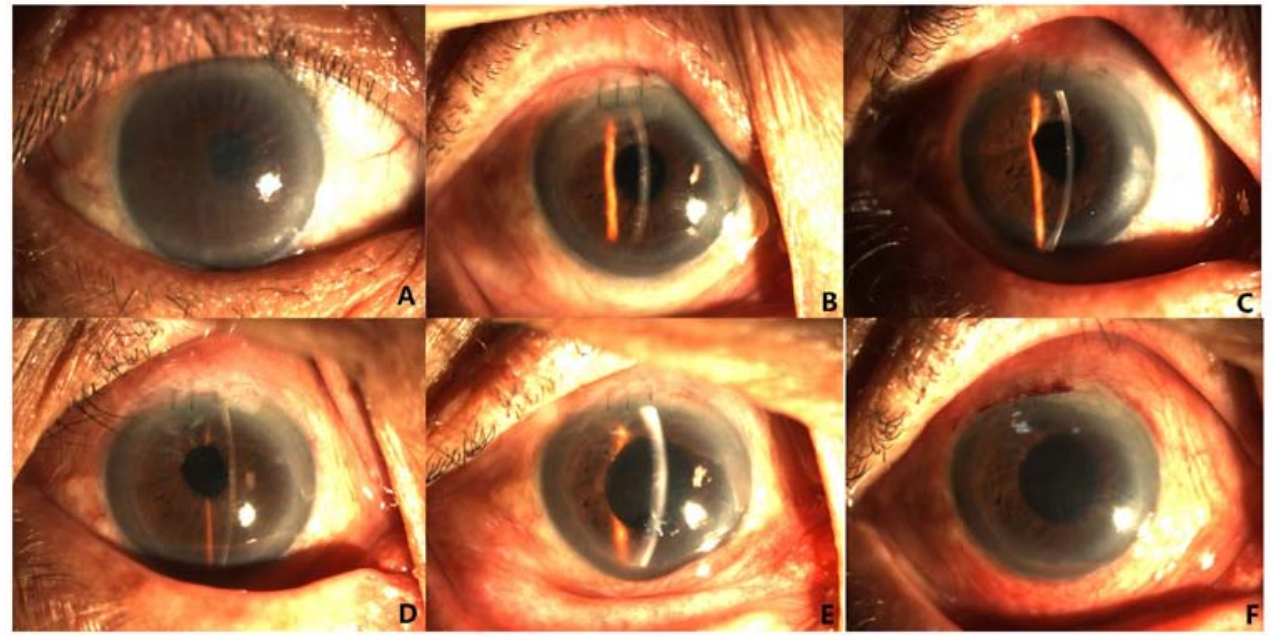

Figure 4. Graft rejection after simplified Descemet stripping endothelial keratoplasty of a 45-year-old male patient. (A) At the preoperative stage, corneal edema and epithelial vesicular changes were present. At (B) 1 month, (C) 3 months and (D) 6 months postoperatively, the corneal graft was smooth and transparent and the depth of the anterior chamber was normal. (E) At 7 months postoperatively, localized corneal epithelial vesicle-like changes and thickened corneal edema were observed. (F) At 8 months postoperatively, the area of corneal graft edema increased after anti-rejection treatment.

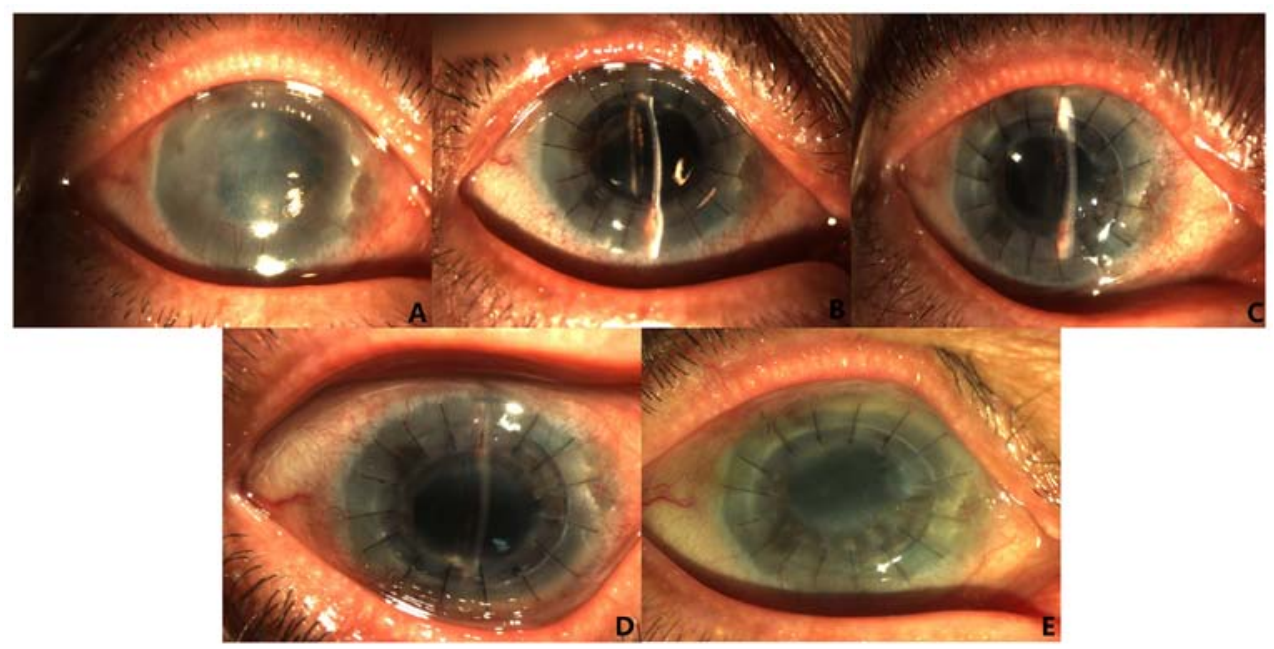

Figure 5. Graft rejection after simplified penetrating keratoplasty of a 53-year-old male patient. (A) At the preoperative stage, corneal edema and epithelial vesicular changes were present. (B) At 1 month postoperatively, the corneal grafts were smooth and transparent, the implants were well aligned with the implant bed and the sutures were in place. (C) At 3 months postoperatively, corneal graft edema and localized corneal epithelial vesicle-like changes were observed. (D) At 6 months postoperatively, the corneal endothelium gradually decompensated after anti-rejection treatment. (E) At 1 year postoperatively, corneal grafts exhibited extensive misty edema and thickening.

sterile air during DSEK to press the graft onto the graft bed. Relevant studies also suggested that the incidence of postoperative glaucoma is closely linked to the history of glaucoma disease prior to surgery $(25,26)$. In the present study, $54.55 \%$ of patients with postoperative glaucoma in the PKP group had glaucoma prior to surgery, while $84.62 \%$ of the patients with postoperative glaucoma in simplified DSEK group had glaucoma prior to surgery. 
During the 1-year follow-up, the incidence of graft rejection in the PKP group was higher than that in the simplified DSEK group, consistent with a previous study (27). The reasons for the lower incidence of postoperative rejection in the DSEK group as compared with that in the PKP group are as follows (28): i) Preservation of the integrity of the host corneal epithelium may reduce the antibody response of the host's immune system to donor alloantigens; ii) the donor tissue does not include the shallow stroma of the cornea, while the donor stroma contains dendritic cells, which serve an important role in host immune activation. Associated studies have indicated that poor postoperative compliance and unauthorized withdrawal or reduction of glucocorticoid eye drops is one of the most important causes of rejection following DSEK $(29,30)$. Glucocorticoid eye drops are currently the major treatment for rejection after corneal transplantation and its effective rate in reversing rejection was reported to be between 39 and $92 \%$ (31).

In the present study, the incidence of postoperative graft infection in the PKP group was $22.22 \%(6 / 27)$ and the treatment failure rate was $66.67 \%$ (4/6). In the simplified DSEK group, 1 eye had secondary corneal ulcers caused by bacterial infection at 1 year after the operation, which was controlled following anti-infective treatment. The incidence of graft infection after PKP was higher than that of simplified DSEK. The possible reasons are as follows: i) PKP reduces the integrity of the cornea and destroys the ocular surface barrier; ii) the innervation of the corneal surface was cut during surgery and postoperative hypoesthesia of the corneal graft became a susceptibility factor; iii) there are numerous sutures of corneal grafts after surgery and loosening or rupture of sutures may easily induce corneal graft infection. It was reported that if interstitial infection and endophthalmitis or advanced keratitis occur early after DSEK (within 3 months after surgery), the consequences may be severe and certain patients require treatment with PKP (21). Therefore, although the incidence of postoperative infection after DSEK is low, the occurrence of infection should also be taken into account and early intervention should be provided.

Patients in the DSEK group were more frequently subjected to combined procedures compared with the PKP group, which certainly impacted the BCVA results. However, corneal endothelial transplantation combined with cataract extraction surgery has gradually become a routine procedure, particularly for elderly patients. After removal of the lens and the placement of the donor IOL, the anterior chamber is deepened, which is conducive to the insertion of endothelial implants and also reduces the risk of postoperative high IOP. Although the number of combined operations in the two groups was not balanced, the degree of BK was equivalent and did not affect the results, therefore allowing for comparison. Furthermore, most patients without combined surgery underwent phacoemulsification surgery or IOL implantation. Therefore, this does not affect the overall results and comparison is possible.

There were certain limitations to the present study. First, selective suture removal was not performed in the present study, which should be performed to achieve optimal vision. In addition, endothelial cell density was not assessed in the study. The small sample size and short follow-up were further limitations of the present study. Therefore, a large randomized controlled study should be performed in the future.
In conclusion, the simplified DSEK technique for the treatment of BK achieved good postoperative outcomes and the complications were similar to those of the conventional DSEK technique. Compared with that in the PKP group, the visual acuity in the simplified DSEK group was significantly better than that of the PKP group during and at the end of the postoperative follow-up. The incidence of postoperative secondary glaucoma, graft rejection and secondary infection was lower in the simplified DSEK than in the PKP group. However, only the difference in the incidence of secondary infection was statistically significant, which may have been due to the small number of cases included and the short follow-up time.

\section{Acknowledgements}

Not applicable.

\section{Funding}

No funding received.

\section{Availability of data and materials}

All data generated or analyzed during this study are included in this published article.

\section{Authors' contributions}

YC and MG conceived the study. YC and MG performed the literature search and writing of the manuscript. SS and QL analyzed and interpreted the data. SW and ZW collected and assembled the data. MG submitted the manuscript and is the corresponding author. All authors read and approved the final manuscript.

\section{Ethics approval and consent to participate}

The present retrospective study was approved by the Institutional Review Board of The General Hospital of Shenyang Military Area Command k(2017)25 (Shenyang, China).

\section{Patient consent for publication}

Not applicable.

\section{Competing interests}

The authors declare that they have no competing interests.

\section{References}

1. Cardascia N, Pastore V, Bini V, Lategola MG and Alessio G: Graft detachment after Descemet's stripping automated endothelial keratoplasty in bullous keratopathy and fuchs dystrophy. Med Hypothesis Discov Innov Ophthalmol 9: 15-22, 2020.

2. Zhang T, Li SW, Chen TH, He JL, Kang YW, Lyu FQ, Ning JH and Liu C: Clinical results of non-Descemet stripping endothelial keratoplasty. Int J Ophthalmol 10: 223-227, 2017.

3. Price MO, Gorovoy M, Price FW Jr, Benetz BA, Menegay HJ and Lass JH: Descemet's stripping automated endothelial keratoplasty: Three-year graft and endothelial cell survival compared with penetrating keratoplasty. Ophthalmology 120: 246-251, 2013. 
4. Acar BT, Akdemir MO and Acar S: Visual acuity and endothelial cell density with respect to the graft thickness in Descemet's stripping automated endothelial keratoplasty: One year results. Int J Ophthalmol 7: 974-979, 2014

5. Phillips PM, Terry MA, Shamie N, Chen ES, Hoar K, Dhoot D, Shah AK, Friend DJ, Rao NK and Davis-Boozer DD: Descemet stripping automated endothelial keratoplasty in eyes with previous trabeculectomy and tube shunt procedures: Intraoperative and early postoperative complications. Cornea 29: 534-540, 2010

6. Maier P, Reinhard T and Cursiefen C: Descemet stripping endothelial keratoplasty-rapid recovery of visual acuity. Dtsch Arztebl Int 110: 365-371, 2013.

7. Price MO and Price FW Jr: Descemet's stripping with endothelial keratoplasty: Comparative outcomes with microkeratome-dissected and manually dissected donor tissue. Ophthalmology 113: 1936-1942, 2006

8. Mehta JS, Por YM, Beuerman RW and Tan DT: Glide insertion technique for donor cornea lenticule during Descemet's stripping automated endothelial keratoplasty. J Cataract Refract Surg 33: 1846-1850, 2007.

9. Ang M, Mehta JS, Lim F, Bose S, Htoon HM and Tan D Endothelial cell loss and graft survival after Descemet's stripping automated endothelial keratoplasty and penetrating keratoplasty. Ophthalmology 119: 2239-2244, 2012.

10. Shinton AJ, Tsatsos M, Konstantopoulos A, Goverdhan S, Elsahn AF, Anderson DF and Hossain P: Impact of graft thickness on visual acuity after Descemet's stripping endothelial keratoplasty. Br J Ophthalmol 96: 246-249, 2012.

11. Price MO, Price FW Jr, Kruse FE, Bachmann BO and Tourtas T: Randomized comparison of topical prednisolone acetate $1 \%$ versus fluorometholone $0.1 \%$ in the first year after descemet membrane endothelial keratoplasty. Cornea 33: 880-886, 2014.

12. Borderie VM, Loriaut $\mathrm{P}$, Bouheraoua $\mathrm{N}$ and Nordmann JP: Incidence of intraocular pressure elevation and glaucoma after lamellar versus full-thickness penetrating keratoplasty. Ophthalmology 123: 1428-1434, 2016.

13. Sandhu S, Petsoglou C, Grigg J and Veillard AS: Elevated intraocular pressure in patients undergoing penetrating keratoplasty and descemet stripping endothelial keratoplasty. J Glaucoma 25: 390-396, 2016

14. Kaleem M, Ridha F, Shwani Z, Swenor B, Goshe J and Singh A Rates of intraocular pressure elevation and use of topical antihypertensive medication after descemet stripping automated endothelial keratoplasty. Cornea 36: 669-674, 2017.

15. Kosker M, Suri K, Duman F, Hammersmith KM, Nagra PK and Rapuano CJ: Long-term outcomes of penetrating keratoplasty and Descemet stripping endothelial keratoplasty for fuchs endothelia dystrophy: Fellow eye comparison. Cornea 32: 1083-1088, 2013.

16. Aldave AJ, Chen JL, Zaman AS, Deng SX and Yu F: Outcomes after DSEK in 101 eyes with previous trabeculectomy and tube shunt implantation. Cornea 33: 223-229, 2014.

17. Ang M, Soh Y, Htoon HM, Mehta JS and Tan D: Five-year graft survival comparing descemet stripping automated endothelial keratoplasty and penetrating keratoplasty. Ophthalmology 123 : $1646-1652,2016$

18. Woo JH, Ang M, Htoon HM and Tan D: Descemet membrane endothelial keratoplasty versus descemet stripping automated endothelial keratoplasty and penetrating keratoplasty. Am J Ophthalmol 207: 288-303, 2019.
19. Kornmann HL and Gedde SJ: Glaucoma management after corneal transplantation surgeries. Curr Opin Ophthalmol 27: 132-139, 2016.

20. Huber KK, Maier AK, Klamann MK, Rottler J, Özlügedik S, Rosenbaum K, Gonnermann J, Winterhalter S and Joussen AM: Glaucoma in penetrating keratoplasty: Risk factors, management and outcome. Graefes Arch Clin Exp Ophthalmol 251: 105-116, 2013.

21. Basak SK and Basak S: Complications and management in Descemet's stripping endothelial keratoplasty: Analysis of consecutive 430 cases. Indian J Ophthalmol 62: 209-218, 2014.

22. Lee WB, Jacobs DS, Musch DC, Kaufman SC, Reinhart WJ and Shtein RM: Descemet's stripping endothelial keratoplasty: Safety and outcomes: A report by the American academy of ophthalmology. Ophthalmology 116: 1818-1830, 2009.

23. Espana EM, Robertson ZM and Huang B: Intraocular pressure changes following Descemet's stripping with endothelial keratoplasty. Graefes Arch Clin Exp Ophthalmol 248: 237-242, 2010.

24. Maier AK, Klamann MK, Torun N, Gonnermann J, Schroeter J, Joussen AM and Rieck P: Intraocular pressure elevation and post-DSEK glaucoma after Descemets stripping endothelial keratoplasty. Graefes Arch Clin Exp Ophthalmol 251: 1191-1198, 2013.

25. Karadag O, Kugu S, Erdogan G, Kandemir B, Eraslan Ozdil S and Dogan OK: Incidence of and risk factors for increased intraocular pressure after penetrating keratoplasty. Cornea 29: 278-282, 2010.

26. Allen MB, Lieu P, Mootha VV, Bowman RW, Petroll WM, Tong L, Kooner KS, Cavanagh HD, Whitson JT and Aggarwal NK: Risk factors for intraocular pressure elevation after descemet stripping automated endothelial keratoplasty. Eye Contact Lens 36: 223-227, 2010.

27. Hjortdal J, Pedersen IB, Bak-Nielsen S and Ivarsen A: Graft rejection and graft failure after penetrating keratoplasty or posterior lamellar keratoplasty for fuchs endothelial dystrophy. Cornea 32: e60-e63, 2013

28. Jordan CS, Price MO, Trespalacios R and Price FW Jr: Graft rejection episodes after Descemet stripping with endothelial keratoplasty: Part one: Clinical signs and symptoms. $\mathrm{Br}$ J Ophthalmol 93: 387-390, 2009.

29. Li JY, Terry MA, Goshe J, Shamie N and Davis-Boozer D Graft rejection after Descemet's stripping automated endothelial keratoplasty: Graft survival and endothelial cell loss. Ophthalmology 119: 90-94, 2012.

30. Allan BD, Terry MA, Price FW Jr, Price MO, Griffin NB and Claesson M: Corneal transplant rejection rate and severity after endothelial keratoplasty. Cornea 26: 1039-1042, 2007.

31. Hashemian MN, Latifi G, Ghaffari R, Ghassemi H, Zarei-Ghanavati M,Mohammadi SF, Yasseri M,Fallah Tafti MR and Tafti ZF: Topical tacrolimus as adjuvant therapy to corticosteroids in acute endothelial graft rejection after penetrating keratoplasty: A randomized controlled trial. Cornea 37: 307-312, 2018.

This work is licensed under a Creative Commons Attribution-NonCommercial-NoDerivatives 4.0 International (CC BY-NC-ND 4.0) License. 